- JOURNAL OF SPACECRAFT AND ROCKETS

Voi. 39, No.'A, July-Augut-2002 (Tentative)

\title{
5 September toctoker \\ Nonlinear Pressurization and Modal Analysis Procedure for Dynamic Modeling of Inflatable Structures
}

\author{
Kurt B. Smalley* \\ Clarkson University, Potsdam, New York 13699

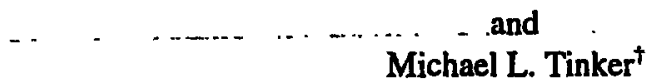 \\ NASA Marshall Space Flight Center, Huntsville, Alabama 35812
}

\begin{abstract}
An introduction and set of guidelines for finite element dynamic modeling of nonrigidized inflatable structures is provided. A two-step approach is presented, involving 1) nonlinear static pressurization of the structure and updating of the stiffness matrix and 2) linear normal modes analysis using the updated stifmess. Advantages of this approach are that it provides physical realism in modeling of pressure stiffening, and it maintains the analytical convenience of a standard linear eigensolution once the stiffmess has been modified. Demonstration of the approach is accomplished through the creation and test verification of an inflated cylinder model using a large commercial finite element code. Good frequency and mode shape comparisons are obtained with test data and previous modeling efforts, verifying the accuracy of the technique. Problems encountered in the application of the approach, as well as their solutions, are discussed in detail.
\end{abstract}

\section{Nomenclature}

$\begin{array}{ll}F_{1}, F_{2} & =\text { element forces in nonlinear static solution, } \mathrm{N} \\ K_{0}, K_{1} & =\text { estimates of tangent stiffness, } \mathrm{N} / \mathrm{mm} \\ P, P_{1}, P_{2} & =\text { applied loads, } \mathrm{N} \\ R_{1}, R_{2} & =\text { unbalanced loads, } \mathrm{N} \\ u & =\text { displacement, } \mathrm{mm} \\ \Delta P_{1}, \Delta P_{2} & =\text { applied load increiments, } \mathrm{N} \\ \Delta U_{0}, \Delta U_{1} & =\text { displacement prediction and correction, } \mathrm{mm}\end{array}$

\section{Introdiction}

I NFLATED cylindrical struts constructed of thin polymer films have considerable practical application and potential for use as components of inflatable concentrator assemblies, antenna structures, and space power systems. Because of their importance, it is of great interest to characterize the dynamic behavior of inflatable cylinders both experimentally and analytically. It is very helpful to take a building-block approach to modeling and understanding inflatable assemblies by first investigating in detail the behavior of components such as struts. The film material used for construction of such cylinders (and the film-pressurized air interaction) is highly nonlinear, with modulus varying as a function of frequency, temperature, and level of excitation. Thus, the primary motivation of tests and analytical modeling efforts was to determine and understand the response of inflatable cylinders for different pressures and boundary conditions. In this investigation, it is assumed that the structure has not yet been (or will not be) rigidized following its inflation.

In recent years, inflatable structures have been the subject of renewed interest for space applications such as communications antennas, solar thermal propulsion, and space solar power. ${ }^{1-6} \mathrm{~A}$ major advantage of using inflatable structures in space is that they are extremely lightweight. Additional obvious advantages include onorbit deployability and related volume savings in the launch configuration. A recent technology demonstrator flight for inflatable structures was the inflatable antenna experiment that was deployed on

\footnotetext{
Received 18 June 2001; revision received 16 January 2002; accepted for publication 28 May 2002.

Copies of this paper may be made for personal or internal use, on condition that the copier pay the $\$ 10.00$ per-copy fee to the Copyright Clearance Center, Inc., 222 Rosewood Drive, Danvers, MA 01923; include the code $0022-4650 / 02 \$ 10.00$ in correspondence with the CCC.

thtudent of Mechanical Engineering. Associate Fellow AIAA.
}

orbit from the Shuttle Orbiter. Although difficulty was encountered in the inflation/deployment phase, the flight was successful overall and provided valuable experience in the use of such structures. ${ }^{1.2}$

The Solar Orbital Transfer Vehicle ${ }^{3}$ is a potential technology demonstrator for solar thermal propulsion. The basic concept behind solar thermal propulsion is to utilize sunlight or solar energy as a means of heating a working fluid (propellant) to provide thrust at increased specific impulse. As described in Ref. 7, thrust is produced by expanding the heated propellant through a nozzle. No combustion occurs, and the thrust level is low. For this reason, solar thermal propulsive systems are mainly applicable for orbital transfer vehicles.

Another technology demonstration concept for solar thermal propulsion is the Solar Thermal Upper Stage (STUS), which is described in Ref. 8. The engine system envisioned for the STUS is designed to utilize hydrogen propellant to produce a thrust level of about $8.9 \mathrm{~N}(2 \mathrm{lbf})$. Two inflatable parabolic collectors could be used that would be rotated and gymballed for focusing sunlight into an absorber cavity. The collectors would be inflated after separation of the upper stage from the launch vehicle.

In Figs. 1 and 2, a prototype inflatable solar concentrator ${ }^{9,10}$ is shown that consists of a torus/lens assembly supported by three struts. This concentrator is constructed of Kapton polyimide film, with epoxy as the primary adhesive for joints. Adhesive tape was also utilized in this concentrator to attach the lens to the torus. The Fresnel lens of such a concentrator assembly would focus sunlight into a collector near the fixed ends of the struts. Solar energy stored in the collector could be utilized to heat a propellant, as described earlier, or for generation of electrical power. The inflatable struts are attached to a base plate or spacecraft by means of three cylindrical appendages. These hollow appendages also allow inflation of the concentrator assembly through air hoses connected at each strut. It can be seen that inflatable cylindrical struts (Fig. 3) are critical components of structural assemblies for practical applications. In view of their importance, structural dynamic and static behavior of typical inflated film struts need to be investigated.

The purpose of the current paper is to present a practical finite element approach, using a commercially available code, that will enable researchers to model the dynamics of nonrigidized inflatable structures accurately. To date, relatively little work has been published on dynamic modeling of inflatables, and most methods presented are either approximate and lacking physical realism, or very laborious in their implementation. First, the paper will give an overview of previous modeling of inflatable cylinders. An introduction is then given for the present method of modeling, which 


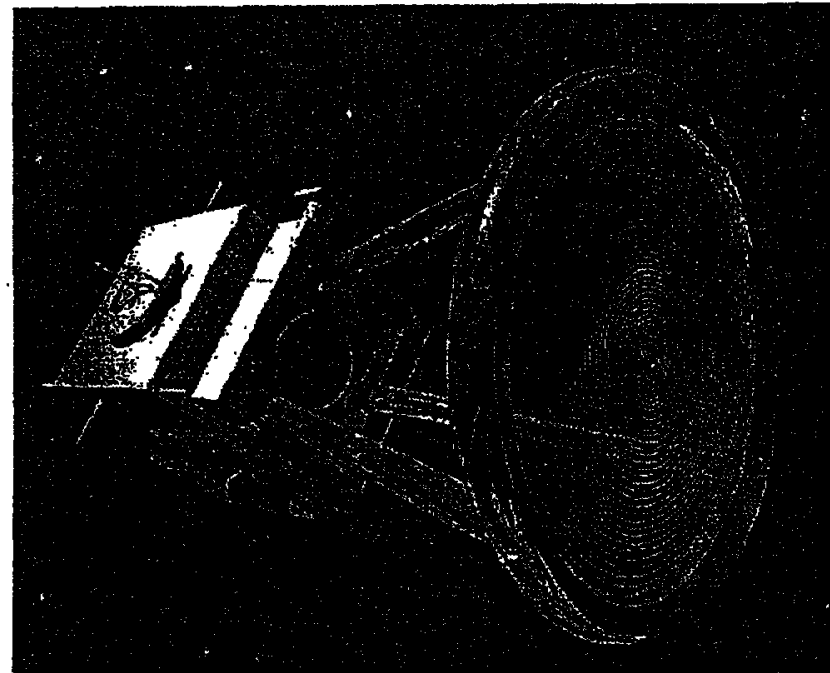

Fig. 1 Concept for solar thermal propulsion system utilizing inflatable components.

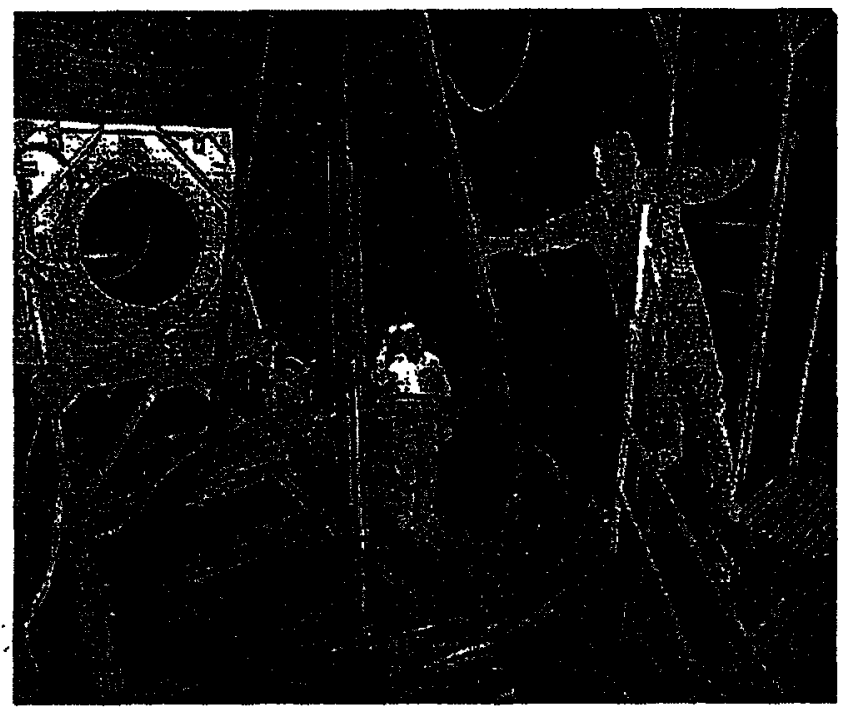

Fig. 2 Prototype solar concentrator in test chamber.

involves a two-step procedure for 1) nonlinear pressurization of the structure and updating of the stiffness matrix and 2) modal analysis using the updated stiffness matrix. The specific mechanics of the method are discussed in parallel with a discussion of their implementation in NASTRAN code. Problems encountered with the method are then discussed, and approaches for their solutions are given. The methodology is verified through the correlation between an analytical model and modal test results of a thin-film strut.

Recommendations are given for the needed advancement of this method and capability to model thin film structures accurately. Finally, conclusions are drawn on the usefulness of the methodology.

\section{Review of Previous Work with Inflated Beamlike Structures}

A number of investigators have considered the use of inflatable cylindrical beams. Perhaps the earliest was Otto, "1 who published ideas for inflated tubular frames for use in structures such as orbiting platforms. A more recent proposed application involves the use of inflatable beam segments to replace solid segments of the Epace

$\therefore$ cqp (Shuttle remote manipulator system and, thus, reduce storage space and inertia of the arm. ${ }^{12}$

Several papers on static structural analysis of inflated cylinders describe different techniques such as linear shell theory and nonlinear and variational methods. ${ }^{13-17}$ Fichter $^{18}$ developed nonlin-

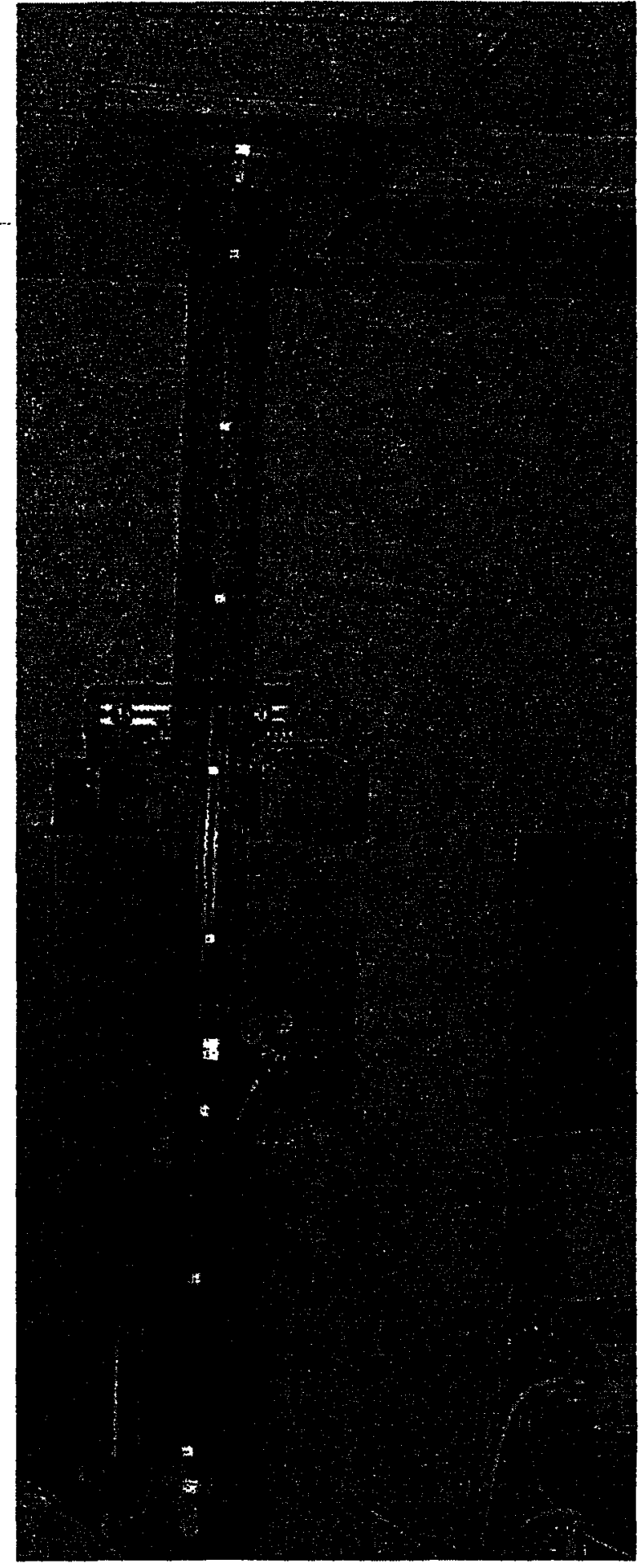

Fig. 3 Inflatable cylindrical strut in cantilever test configuration.

with internal pressure and axial force set to zero, reduced to the Timoshenko beam equations. Comer and Levy, ${ }^{19}$ Webber, ${ }^{20}$ and Main et al. ${ }^{21}$ modeled an inflated beam using an Euler-Bernoulli approach, but with modified material longitudinal modulus and beam moment of inertia. Wrinkling behavior of the material was accounted for by the assumption of no load-carrying capability when longitudinal stress in the beam cross section reached zero.

Additional work of significance involves rigidization of inflated beam structures. One proposed concept is the use of injected foam that fills the cylindrical beam cross section, subsequently hardens, and, thus, rigidizes the structure. This approach is discussed in Ref. 22. 
could be utilized in approximating lower-order frequencies of inflatable beams. Main et al. wrote a very significant paper describing results of modal tests of inflated cantilever beams and the determination of effective material properties. ${ }^{24}$ Changes in material properties for different pressures were also discussed, and the beam model was used in a more complex structure. The paper demonstrated that conventional finite element analysis packages could be very useful in the analysis of complex inflatable structures.

Finally, Refs. 25-27 describe closed-form and finite element beam representations of inflatable cylinder dynamics, along with shell-element models for comparison. It was found that frequencydependent modulus, or bending stiffness, was required for the beamelement models to represent the dynamic test results adequately. Shell models performed reasonably well with linear material properties. Note, however, that the film modulus was varied in modal analyses of the shell-element models of Refs. 25-27 to approximate the effects of pressure and still allow for linear eigensolutions. Pressure was included directly only in frequency response analyses. A more realistic and satisfying approach is to include pressure loading directly in the modal analysis process. Such an approach is investigated in this paper and is described in the following sections.

\section{Methodology Overview}

The purpose of this investigation was to verify a procedure for dynamic modal analysis of nonrigidized inflatable structures in NASTRAN that accounts for the increase of structural stiffness due to internal pressure. In this study, the MacNeal-Schwendler Corporation (MSC) version of the finite element code was utilized. ${ }^{28}$ Ordinary eigenvalue analysis in MSCNASTRAN does not allow for the effects of loads applied on a model because modal characterization of a structure is by definition free from active loads. However, in the case of inflated (nonrigidized) structures, bending stiffness is mainly due to internal pressure. Therefore, a standard modal analysis that does not account for internal pressure loads will give incorrect results. To solve this problem, the proper stiffness matrix for the pressurized structure can be generated in a nonlinear static analysis and then imported into a linear. eigenvalue analysis. Of course, modeling of rigidized inflatables is considerably more straightforward and may not require a nonlinear approach.

\section{NASTRAN Nonlinear Static Analysis}

The key to this methodology, and the most difficult step, is the nonlinear static analysis. To understand more fully the problems that arise during this procedure, and the reasons their solutions are successful, an understanding of the nonlinear static analysis procedure (SOL 106) in NASTRAN is necessary. This procedure provides the ability to handle geometric nonlinearities, kinematic nonlinearities, follower forces, large displacements, displacement-variable loads, material nonlinearity, nonlinear stiffness relationships, buckling analysis, contact analysis, and variable boundary conditions. In this paper, only the characteristics of the procedure that are applicable to modeling of inflatable structures will be discussed. These capabilities are the stiffness updating and follower forces schemes, including their iteration and convergence procedures.

The nonlinear static analysis utilized in this study involved the updating of the stiffness matrix throughout the procedure over a nonlinear curve. NASTRAN is able to do this through its loaddisplacement iteration process, shown conceptually in Fig. 4. This process involves initially applying a predetermined fraction of the total load on the structure. Through application of this load, and the initial geometry and property values of the loaded elements, initial values for the displacement and elemental forces are obtained. When the element nodal (edge) forces and the applied load are not in equilibrium, the iteration is repeated with updated values for the displacement and element forces. These values are calculated through the change in displacement due to the difference between the applied load and elemental load. This cycle is repeated until equilibrium is achieved. The point at which equilibrium occurs is called the convergence point. It is at this point that the stiffness matrix is updated from the last iterated displacement and applied load. After convergence of the initial applied load, the analysis advances and applies a greater fraction of the combined load. A solu-

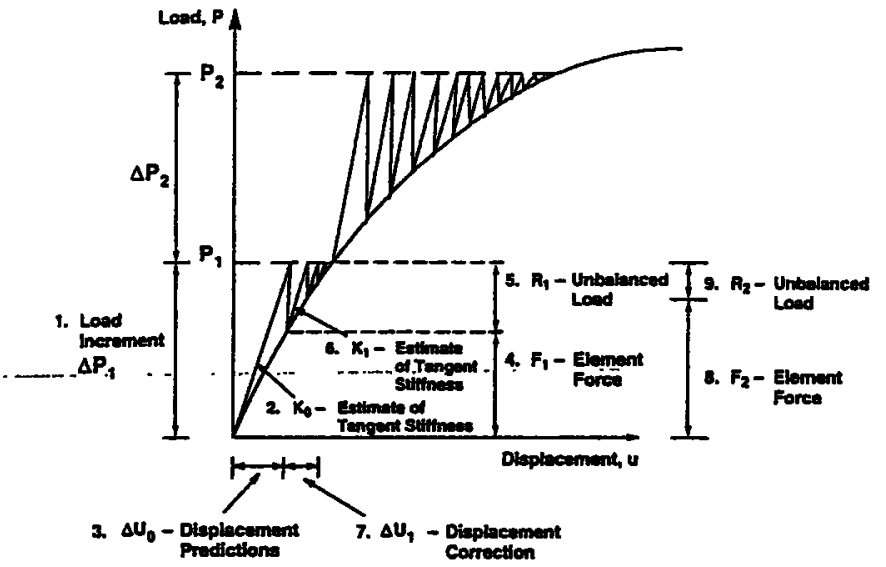

Fig. 4 Basic concept of nonlinear static solution procedure utilized in MSCNASTRAN. (Copyright 191994 by MacNeal-Schwendler Corporation Reprinted by permission.)

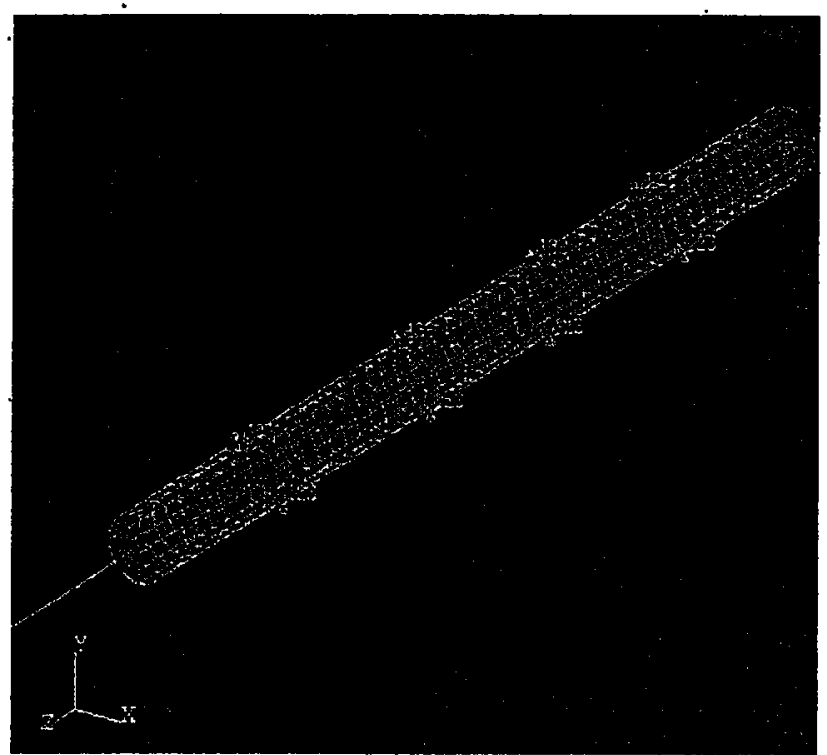

Fig. 5 Example artificial constraint points for pressurization of freefree strut.

tion is obtained when convergence occurs for the entire load. The NASTRAN code has several stiffness updating schemes, iteration and advancing schemes, and convergence criteria, ${ }^{28}$ and the process illustrated in Fig. 4 can vary considerably depending on the choice of solution parameters. The manipulation and use of these analysis schemes and parameters present the greatest challenge in use of this methodology.

For the thin-film cylinders investigated in this study, convergence of the NASTRAN nonlinear pressurization solution was difficult to obtain because the thin material tended to have large displacements in the initial iterations of the analysis. It was found that if the model could be stabilized through the first several iterations (to reach the point where changes in nodal displacements between analysis steps became smaller) the solution proceeded easily to convergence. The authors found that one way to stabilize the nonlinear solution was to constrain the models artificially. For example, artificial constraints were placed at points on the film surface along the length of a freefree pressurized cylinder (Fig. 5), at a sufficient number of locations? in to suppress the largest displacements. Only the rotational degrees of freedom were allowed at these constrained nodes. This method was found to be workable for the analysis, andzin generabzor this on methodology, particularly for large structures, points on the thinfilm surface must either be artificially constrained as illustrated in Fig. 5, or artificially stiffened in the first analysis steps. Models for large complex structures may require a trial-and-error approach of observing the locations of largest displacements in the initial steps 


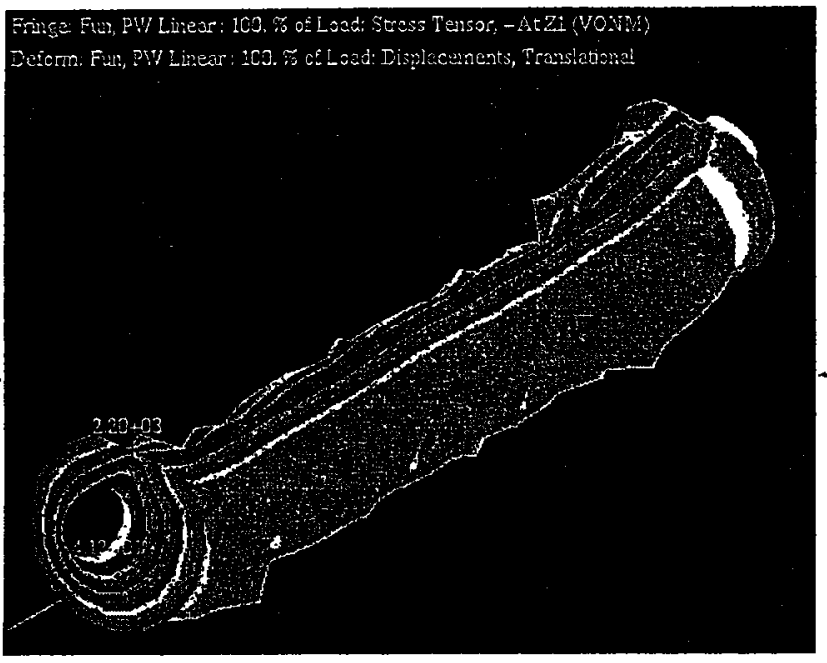

Fig. 6 Example deformed shape of inflated strut with artificial constraints.

of the pressurization analysis and then applying artificial constraints at those locations.

It was determined for the cylinder models that the artificial constraints only marginally changed the final values of the updated stiffness matrix, even though the constraints modified the displaced shape of the example pressurized model discussed in the preceding paragraph (Fig. 6). The spikes evident on the surface of the model result from the tendency of the film material to have large displacements during pressurization, combined with the effects of the artificial constraints. Apparently, the stiffness of the thin-film elements is controlled largely by the magnitude of the displacements and not by the direction of them. Even though the artificial constraints altered the geometry of the elements, the strain and stress of those elements remained mostly unchanged and the stiffness matrix was only slightly altered.

The minimal effect of artificial surface constraints on model stiffness can be further explained by noting that the magnitudes of the elemental normal forces rely greatly on the curvature of that element. The greater the angle of curvature, the larger the component of the tensile force that can react against the applied load. Left alone, an element will displace rather than increase its currvature, causing convergence problems in NASTRAN due to large displacements. The artificial constraints allowed the elements to increase their rate of curvature in relation to the load applied, rather than to displace as a rigid body. This increase in curvature is still translated into strain, though in a different direction, allowing stress and, thus, updated stiffness to remain mostly unaltered in comparison to an unconstrained pressurization. Of course, if two adjacent nodes on an element were artificially constrained, the stiffness matrix would be significantly modified.

Once the artificial constraints are in place, the nonlinear static analysis is performed. In this analysis, NASTRAN solution SOL 106 allows for the manipulation of the parameters and solution schemes in the model description (bulk data section). ${ }^{28}$ Many convergence problems can be overcome through the manipulation of these parameters. The authors found that for this study it was adequate to alter only the convergence criteria and their margins of error and to use default values for other analysis parameters. Further investigation into the manipulation of other parameters is certainly needed. NASTRAN has the option of choosing between displacement, energy, or load as the criterion from which it will base its convergence. The authors found it to be useful to activate all three to allow analysis advancement from whichever criterion converges first. It was also found useful to increase the error tolerances for these criteria from their default values, though care must be taken in doing so. Solution accuracy is, thus, traded off with the increased potential for convergence. It was also discovered that too large an error tolerance was just as fatal as one too small for achieving convergence. It is thought that too large a tolerance takes later iterations away from the convergence point.
An alternative to the use of artificial constraints is the application of artificial stiffness to the model during the initial load iterations. This can be done in automated fashion in MSC/NASTRAN through the KDIAG parameter, ${ }^{28}$ which results in the addition of a user-prescribed stiffness value (value of KDIAG) to the diagonal terms of the model stiffness matrix. The additional stiffness is applied only during the earliest iterations of the procedure to get the nonlinear solver started and is then removed automatically by NASTRAN once the model is stabilized. Obviously, the automated nature of this approach makes it attractive, and it is the method recommended by.MSC..However, there appears to be a potentially significant disadvantage to the KDIAG approach for very flexible thin-walled structures. The procedure was not utilized in this study, but it was discovered during modeling of a large inflatable optical membrane that the analysis results were sensitive to the value of KDIAG chosen by the analyst. As a result, trial and error assessments of the value to be added to the diagonal stiffness terms may be necessary, thus, largely negating the advantages of the KDIAG approach.

\section{NASTRAN Modal Restart}

On convergence of the nonlinear static analysis, a modal restart is performed (Fig. 7). This restart is simply the second stage of the analysis procedure, which is activated through the RESTART command in the executive control section ${ }^{28}$ of the file. This command imports the MASTER file (containing information about the model and results of the first run) from the nonlinear analysis and claims it as its own. From this MASTER file, the restart uses the original geometry of the model and the stiffness matrix from a chosen iteration. In the bulk data section of the restart run, the desired iteration of the nonlinear static analysis stiffness update can be chosen. Also, at this point in the analysis, the artificial constraints discussed earlier must be removed by deleting the appropriate lines in the sorted bulk data from the nonlinear analysis. The procedure for removing the constraints is demonstrated in the last several lines of Fig. 7, where the appropriate line numbers to be deleted were found in the sorted bulk data echo in the nonlinear f06 (results) file. Remaining

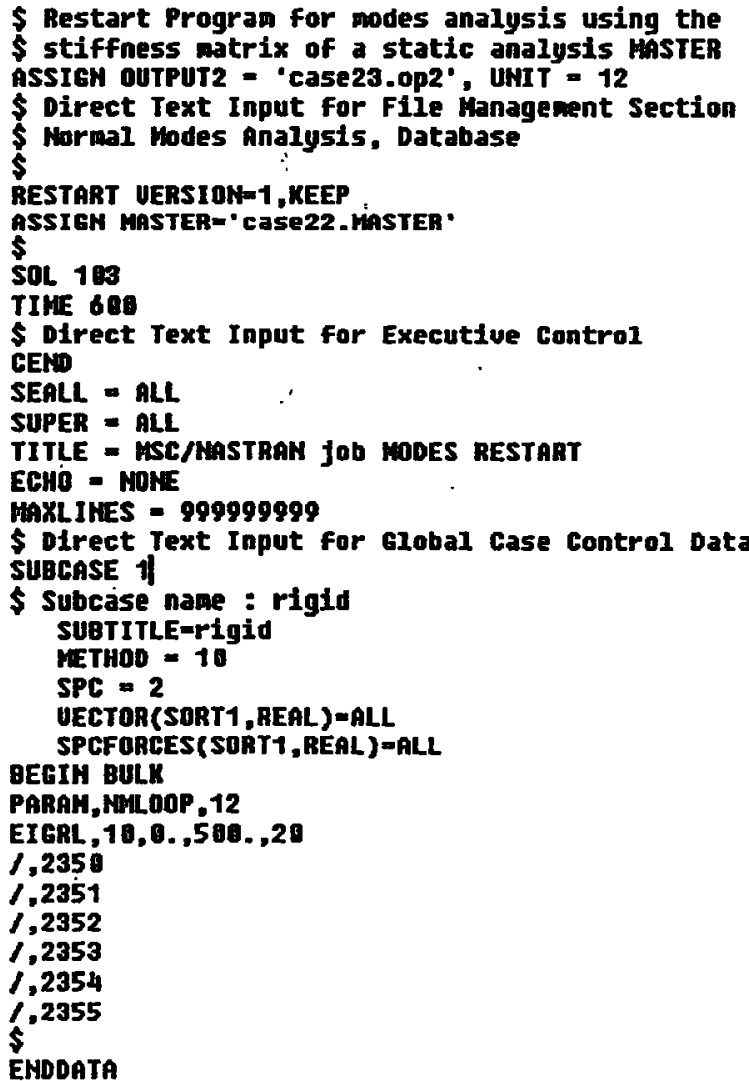

Fig. 7 NASTRAN runstream for modal solution utilizing updated stiffness matrix from nonlinear pressure solution. 
sections of the restart are organized as an ordinary SOL 103 eigenvalue analysis. Within the restart command, it is useful to use the KEEP command for retaining the old MASTER file for later use. Reference 28 provides an overview of the capability and file strucure for the NASTRAN finite element code, and additional references provide further information for dynamic modeling ${ }^{29}$ and nonlinear analysis. ${ }^{30}$ For the interested reader, the various commands and entries for the restart file in Fig. 7 are described in detail in Ref. 28.

\section{Application of Methodology to Dynamics of Inflated Cylindrical Beams}

The solution procedure described in the preceding two sections was performed and ultimately verified through the modeling of thinfilm inflatable struts characterized earlier. A typical inflated cylindrical beam or strut of the type investigated in this paper is shown in Fig. 3. The beams studied are $2.44 \mathrm{~m}$ ( $8 \mathrm{ft}$ ) long with $15.2-\mathrm{cm}$ (6-in.) diameter, are constructed of Kapton polyimide film, and are sealed at the ends using styrofoam plugs. Openings in the plugs provided the means for inserting air hoses to inflate the structure to desired pressure. Epoxy was used to bond the foam plug to the polyimide film walls of the cylinder.

The beams were constructed by overlapping the edges of the polyimide sheet and placing a thin layer of epoxy adhesive along the overlapped area to form a joint running the length of the beam. In a cross-sectional view, the bonded joint looks like a sandwich, with the epoxy layer between two layers of Kapton film. Of course, the bonded region has much higher stiffness than a nominal polyimide section of cylinder wall, and this had to be accounted for in modeling of the struts. The stiffness of the joint appears to be dominated by the epoxy layer rather than the polyimide material, partially due to the greater thickness of epoxy. Polyimide film of $51 \mu \mathrm{m}(0.002$ in. or 2 mil) thickness was utilized, and the epoxy layer was typically $255 \mu \mathrm{m}(0.01 \mathrm{in}$. or $10 \mathrm{mil})$ thick.

Quadrilateral shell elements were used to model the strut film material. Nonstructural masses were added to these elements to compensate for the mass of air within the structure. A single line of elements along the length of the strut was thickened to $293 \mu \mathrm{m}$ (11.5 mil) to compensate for the overlap and adhesive along the seam. Solid elements were used for the end plugs.

Once the strut model was completed, the first step in the modal analysis procedure was to constrain the model (artificially in the case of free-free boundary conditions) and perform a nonlinear static pressurization using NASTRAN SOL 106. The constraints were placed on the relatively stiff foam end plug because both cantilever and free-free configurations were to be investigated. Results of the nonlinear analysis appear very reasonable, as shown in Fig. 8. The effect of the seam, with its higher stiffness due to the film overlap and adhesive, is observed in the "hotdog" shape of the strut with the free end bending upward. During the SOL 106 run, the model stiffness matrix was updated to capture the pressure stiffening effect.
The second step in the procedure was to run a linear modal analysis using the updated stiffness matrix. For the free-free cases, the artificial constraints on the end plug were removed before initiating the eigensolution. Figure 7 shows the NASTRAN modal restart runstream used for the free-free configurations of the cylindrical beam. It is noted that, for this analysis, artificial constraints were not placed on the film surface, as was done for the example in Figs. 5 and 6. However, for large inflatable structures, it appears to be necessary to constrain the film at points of largest displacement during pressurization.

The results of analysis using the present methodology compared to modal test data ${ }^{31}$ and previous modeling results from Refs. $25-27$ can be seen in Table" 1 , where frequencies for the lowest bending modes are shown for each case. For all frequencies in Table 1, the mode shapes were classical beam bending-type modes. For example, Fig. 9 shows the second mode shape for the free-free conditions and 9.7-kPa (1.4-psi) pressure. Free-free and cantilever configurations were tested and modeled at multiple pressure levels for each boundary condition. As can be seen in Table 1, the free-free results fell within an acceptable margin of 6\% frequency error in comparison to test frequencies. Furthermore, the free-free analytical frequencies obtained using the present method are considerably more. accurate than the results from Refs. 25-27. Results of two models from Refs. 25-27 are given in Table 1, a linear shell-element model in which the effects of pressure were represented by increasing the film modulus and a nonlinear shell-element model with frequencydependent modulus and directly applied pressure. For the nonlinear shell model, the modal data were extracted from NASTRAN frequency response results in a rather laborious process.

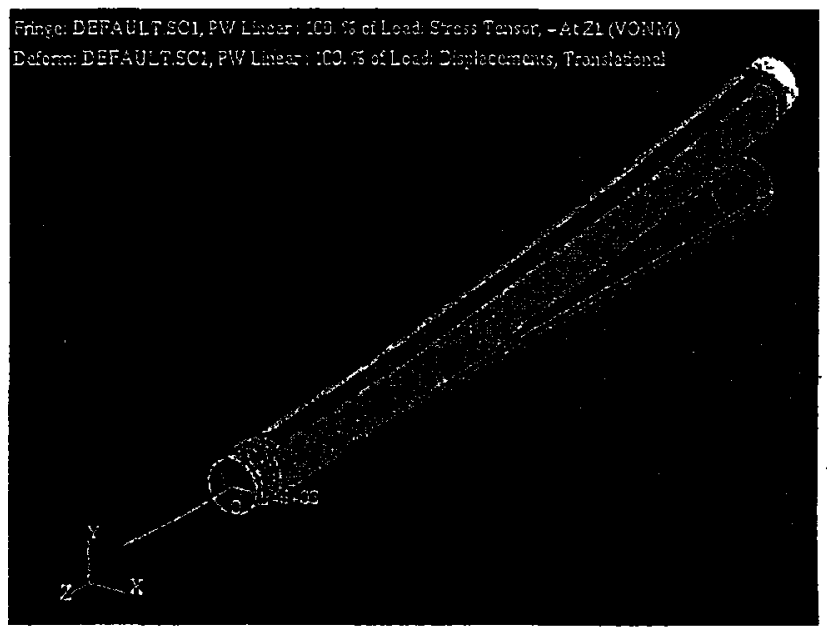

Fig. 8 Deformed shape of pressurized strut with end constraints.

Table 1 Comparison of analytical and experimental modal results for free-free and cantilever boundary conditions and various internal pressures

\begin{tabular}{|c|c|c|c|c|c|c|c|}
\hline \multirow[b]{2}{*}{ Mode } & \multirow[b]{2}{*}{ Experiment frequency, $\mathrm{Hz}$} & \multicolumn{2}{|c|}{ Present method } & \multicolumn{2}{|c|}{ Refs. 25-27 linear } & \multicolumn{2}{|c|}{-Refs. $25-27$ nonlinear } \\
\hline & & Frequency, $\mathrm{Hz}$ & \% error & Frequency, $\mathrm{Hz}$ & \% error & Frequency, $\mathrm{Hz}$ & \% error \\
\hline \multicolumn{8}{|c|}{ Free-Free, $5.2 \mathrm{kPa}$} \\
\hline 1 & 24.30 & 22.85 & $\begin{array}{r}5.97 \\
553\end{array}$ & $\begin{array}{l}22.93 \\
8078\end{array}$ & $\begin{array}{r}5.64 \\
-1251\end{array}$ & 26.50 & -9.05 \\
\hline 2 & 71.80 & $6 / .83$ & $\begin{array}{r}5.53 \\
\text {-Free } 97\end{array}$ & 80.18 & -12.31 & 01.40 & 0.13 \\
\hline $\begin{array}{l}1 \\
2\end{array}$ & $\begin{array}{l}24.60 \\
71.40\end{array}$ & $\begin{array}{l}25.30 \\
70.10\end{array}$ & $\begin{array}{c}-2.85 \\
1.82\end{array}$ & $\begin{array}{l}23.05 \\
81.20\end{array}$ & $\begin{array}{r}6.30 \\
-13.73\end{array}$ & $\begin{array}{l}26.20 \\
68.90\end{array}$ & $\begin{array}{r}-6.50 \\
3.50\end{array}$ \\
\hline \multicolumn{8}{|c|}{ Cantilever, $3.5 \mathrm{kPa}$} \\
\hline 1 & 2.78 & 3.61 & -29.86 & 2.18 & 21.58 & 2.90 & -4.32 \\
\hline 2 & 25.33 & 26.69 & -5.37 & 28.78 & -13.62 & 24.10 & 4.86 \\
\hline 3 & 78.73 & 75.00 & 4.74 & 85.83 & -9.02 & 67.20 & 14.64 \\
\hline \multicolumn{8}{|c|}{ Cuntilever, $6.9 \mathrm{kPa}$} \\
\hline 1 & 2.81 & 4.44 & -58.01 & 2.19 & 22.06 & 2.90 & -3.20 \\
\hline 2 & 25.10 & 28.22 & -12.43 & 28.91 & -15.18 & 23.90 & 4.78 \\
\hline 3 & 79.04 & 76.58 & 3.11 & 86.21 & -9.07 & 67.10 & 15.11 \\
\hline
\end{tabular}




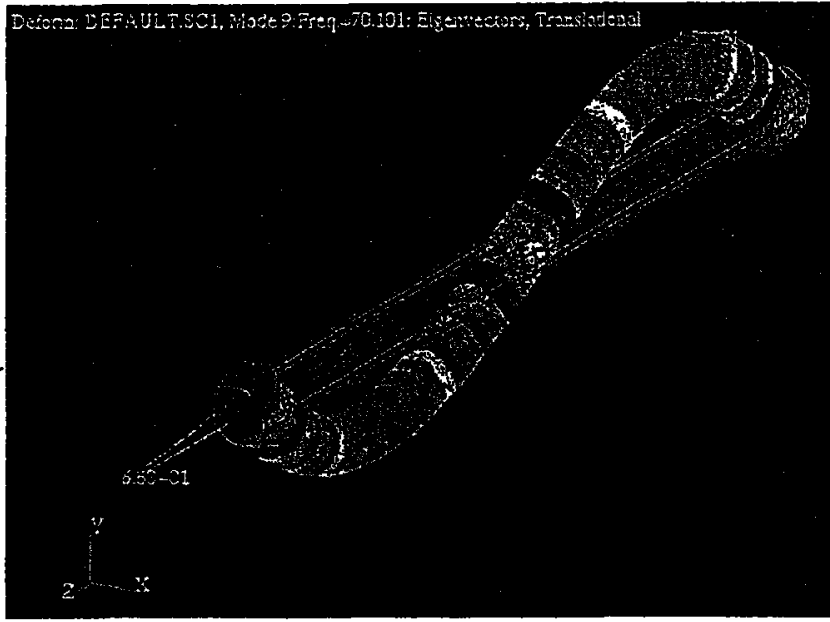

Fig. 9 Second bending mode for tree-free inflated strut, pressure $=9.7 \mathrm{kPa}(1.4 \mathrm{psi})$.

Results for the present methodology in Table 1 for the cantilever arrangement show good model-to-test agreement for the second and third bending modes, but large frequency errors are seen for the fundamental modes. It is possible that these errors in the fundamental frequencies are due to film wrinkling effects near the fixed end of the strut that were not taken into account in the finite element model. Important studies of membrane wrinkling behavior, including cantilever infiated cylinder examples, are described in Refs. 32 and 33. Errors in higher-order mode shapes are possibly due to nonlinear elastic modulus as a function of frequency. Note that geometric nonlinearity (film stretching and stiffening due to internal pressure) was taken into account in modeling efforts described in this paper, but the use of nonlinear material properties in the present methodology also needs to be investigated. With the exception of the fundamental cantilever frequencies, better overall accuracy was achieved with the nonlinear pressurization and linear modal approach than was obtained in Refs. 25-27.

Numerical accuracy of the finite element modeling procedure investigated in this study was verified not only by comparison to test results, but also by comparison to previous finite element modeling efforts and Euler-Bernoulli beam solutions (for the same inflatable strut described in this paper) documented in Refs. 25-27. Furthermore, and perhaps more importantly, because cylindrical beams were modeled, the bending modes were expected and observed to be classical free-free and cantilever beam modes. Prediction of the correct mode shapes by the models developed for this study, as illustrated by Fig. 9, provided tremendous overall confidence in the numerical procedure. Thus, it becomes a matter of verifying numerical accuracy for the model frequencies: Shell models in Ref. 26 had approximately 2000 quadrilateral elements. In the current study, fewer quadrilateral elements (832) were utilized, but it can be concluded from the comparison with Refs. 25-27 and test data in Table 1, particularly for frequencies of the free-free cases, that a sufficient number of elements were used.

Where the use of artificial constraints in the NASTRAN nonlinear static solution is concerned, it was found in this study and related investigations ${ }^{34,35}$ that the locations chosen and the degrees of freedom constrained could be varied without significant effect on the frequencies and mode shapes. Stress distributions and overall displaced shapes obtained in the nonlinear analysis also appeared generally reasonable.

Accuracy of test data was ensured through a number of procedures, ${ }^{31}$ including calibration assessments for the laser vibrometer, load cell, impact hammer, and signal conditioner; functional testing of the test setup; linearity checks for response functions; and visual inspection of instrumentation for verifying proper locations and orientation. Comparison of test frequencies for different pressure levels in the struts also provides a valuable means for assuring consistency and accuracy in the modal data. Test implementation of the desired cantilever boundary conditions was accomplished through the use of a metal clamp attached to the foam plug at the upper end of the strut (Fig. 3). Free-free conditions were achieved through the use of a flexible rubber cord at the upper end. More detailed information on the test configurations and procedures can be found in Refs. 26 and 31 .

Note that the analytical modal results in Table 1 were obtained in the finite element models without the manipulation of the original element properties. That is, the manufacturer's modulus, density, and Poisson's ratio data were used for the polyimide film, which provides a high level of confidence in the solution approach and its ability to predict dynamic characteristics of inflatable structures. This methodology has alsö been implemented successfully in more sophisticated NASTRAN models, which are described in Refs. 34 and 35. Positive results from these larger and more complex models furthered the confidence of the authors in the usefulness and versatility of the procedure. Model-to-test comparisons shown in this paper are only for bending motions of the struts and do not involve the shell behavior. Analytical and experimental comparison of shell modes was not made because the modal test procedure was limited in its ability to identify shell modes.

Important and independent work involving dynamic modeling of an inflatable torus was done in approximately the same time frame as this effort. ${ }^{36}$ The same general approach presented in this paper (static pressurization and subsequent modal analysis using the updated stiffness) was used in Ref. 36 with good results, providing further verification that this approach is proper and accurate for modeling thin-film inflatable structures.

\section{Advantages and Limitations of the Procedure for Modeling Thin-Film Inflatable Structures}

An obvious major advantage of the approach described in this paper is the physical realism achieved by accounting for the internal pressure directly in modal analysis and by accurately modeling the film stiffening due to pressure. Also, this methodology provides the convenience of linear modal analysis in NASTRAN as the second step in the process. The work described in this paper is an extension of previous research described in Refs. 25-27, where for NASTRAN modal solutions the pressure stiffening effect was included indirectly by modifying the strut film modulus. Pressure was modeled directly in Refs. 25-27 only for frequency response analysis incorporating nonlinear modulus, and modal properties were then obtained in a rather painstaking effort from the response functions. The current approach is considerably more convenient for the analyst.

Apparent limitations in this methodology were found throughout the course of the investigation. The small thickness of the inflatable structure material is apparently the primary factor causing difficulty in the use of this methodology. In simple terms, very thin inflatable structures rely on internal pressure for bending stiffness of the membrane. The smaller the thickness of the pressurized structure, the larger the nodal displacements in the initial load steps, making it difficult to obtain a stable analysis. It is also known that elemental forces calculated and applied during the NASTRAN iteration process are a function of the volume of each element. Decreasing the thickness of the film, and, thus, the volume of the elements and the elemental forces applied in each load step, causes a greater number of iterations to be needed for convergence, decreasing the likelihood of NASTRAN reaching a solution. Parameter studies for large inflatable structures have shown that increasing the material thickness generally eliminates the convergence problems discussed in this section.

The angles between very thin adjacent elements also affect the convergence of the nonlinear pressurization solution. This is an important factor, because the element tensile forces, which balance the pressure loads and are proportional to the angles between adjacent elements, impart transverse stiffness to the membrane and, thus, stabilize the numerical solution. The initial element geometries, of course, do not have curvature. Thus, the initial elemental forces that balance pressure loads are not due to the curvature, but rather the angle between adjacent elements. Examining a group of elements as a free-body diagram (Fig. 10), it becomes apparent that the forces reacting to the applied pressure load come from the components of the element edge forces that are parallel and opposite to the pressure vector. These forces are applied to the center element 


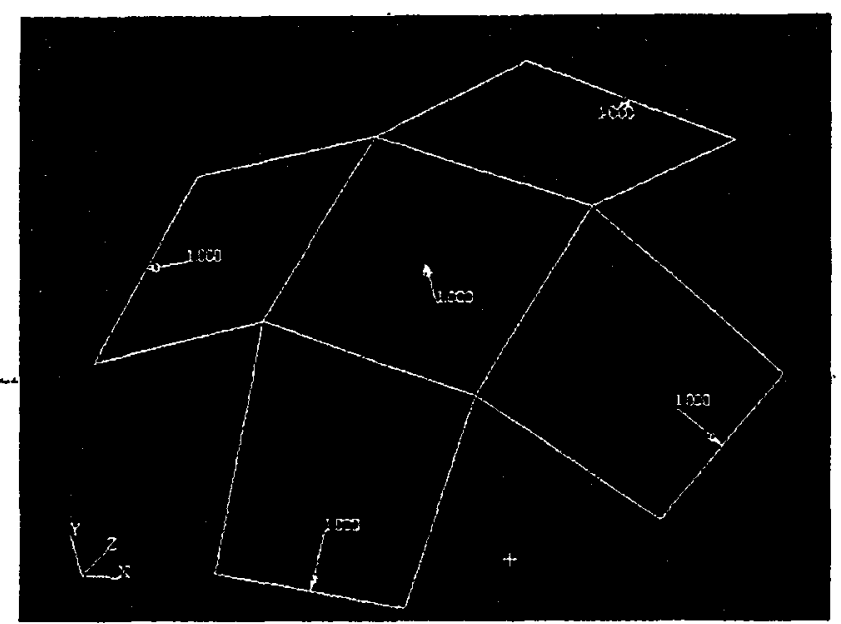

Fig 10 Quadrilateral plate element deformation under pressure loading.

nodes through the surrounding elements. The smaller the angle between adjacent elements, the smaller the force components to resist the pressure, and, thus, initial displacements become larger and retard convergence of the solution. Two difficulties related to small angles between adjacent elements are discussed in the following paragraphs.

The use of a very fine mesh was found to affect convergence adversely in this particular analysis. Not only does a finer mesh complicate the model and increase the computational run time, it also decreases the angles between elements along the curvature of the film. Therefore, a greater number of artificial constraints are needed to provide equilibrium for the elements in early stages of loading. Because of this phenomenon, a coarser mesh may be advantageous, though this observation has not been proven in general for very thin pressurized structures. The fineness of the mesh may not be an issue if the artificial applied stiffness approach (KDIAG parameter discussed in the "Methodology Overview" section) is utilized.

Greater difficulty has been encountered in obtaining numerically stable solutions for geometrically larger thin-film structures ${ }^{35}$ compared to smaller structures. It appears that important parameters are the ratios of material thickness to major dimensions such as length or diameter. Decreases in the angles between adjacent elements and an increase in the'number of elements needed to characterize the structure properly are results of a geometrically larger model. As described in this section, both of these conditions can decrease the ability of NASTRAN to converge on a solution in its nonlinear analysis.

Finally, note that NASTRAN is a large, general-purpose finite element code that is not optimized for nonlinear analysis. The required use of artificial constraints or artificial stiffness as described in this paper clearly points out the difficulty that can be encountered in using general-purpose finite element codes for inflatable structures modeling. Finite element packages more suitable for nonlinear analysis should be investigated, or, if necessary, developed, to make the modeling of thin inflatable structures more convenient for analysts. However, it has been clearly shown in this study that accurate dynamic results can be obtained for inflatable structures using commercially available finite element codes.

\section{Summary}

The investigation and verification of a two-stage finite element procedure for determining the natural modes and frequencies of very thin inflatable structures has been described in this paper. Geometric nonlinear static analysis, which accounts directly for pressure stiffening of the model and results in an updated stiffness matrix, is the first step in the procedure. Subsequently, a linear modal analysis is conducted using the modified stiffness matrix from the first step. Obvious advantages of this approach are that it provides physical realism in modeling of pressure stiffening and that it maintains the analytical convenience of a standard eigensolution once the stiffness has been modified.
Though not investigated in this paper, material nonlinearity can also be accommodated in the first stage of this approach, and the subsequent linear modal analysis can be done, avoiding a painstaking process of determining the modal properties from a nonlinear NASTRAN frequency response analysis. Although good results have been shown in this paper using only the geometric nonlinear capability of the finite element code, use of nonlinear material properties in this procedure should be a subject of future research.

The purpose of this investigation was to verify the usefulness of the methodology through the creation of a working and accurate dynamic-model-of-an-inftated-thin film cylinder, and this has been accomplished. This work has contributed to the understanding of appropriate finite element modeling procedures for inflatable structures, and should help to advance the technology of inflatable space systems.

\section{Acknowledgment}

The inflatable cylindrical strut characterized in this investigation was manufactured by United Applied Technologies, Inc., of Huntsville, Alabama.

\section{References}

'Freeland, R. E., Bilyeu, G. D., and Veal, G. R., "Development of Flight Hardware for a Large, Inflatable-Deployable Antenna Experiment," Acta Astronautica, Vol. 38, Nos. 4-8, 1996, pp. 251-260.

${ }^{2}$ Freeland, R. E., and Veal, G. R., "Significance of the Inflatable Antenna Experiment Technology," Proceedings of the 39th Structures, Structural Dynamics, and Materials Conference, AIAA, Reston, VA, 1998, pp. 27892796.

${ }^{3}$ Pearson, J., Gierow, P., and Lester, D., "Near-Term In-Space Demonstration of an Infiatable Concentrator," AIAA Paper 99-1073, Jan. 1999.

${ }^{4}$ Mankins, J. C., "Space Solar Power. An Assessment of Challenges and Progress," Journal of Aerospace Engineering, Vol. 14, No. 2, 2001, pp. 4651.

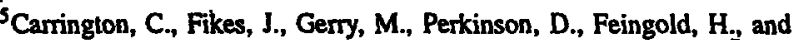
Olds, J., "Abacus/Reflector and Integrated Symmetrical Concentratof Concepts for Space Solar Power Collection and Transmission," AlAA Paper 2000-3067, July 2000.

${ }^{6}$ Grossman, G., and Wiliams, G., "Inflatable Concentrators for Solar Propulsion and Dynamic Space Power," Journal of Solar Energy Engineering, Vol. 112, No. 4, 1990, pp. 229-236.

${ }^{7}$ Stewart, J. F., and Martin, J. A." "Dual Fuel Solar Thermal Stage: Ideal Analysis," Journal of Spacecraft and Rockets, Vol. 33, No. 5, 1996, pp. 752754.

${ }^{8} \mathrm{Hawk}$ C. W., and Adams, A. M., "Conceptual Design of a Solar Thermal Upper Stage (STUS) Flight Experiment," AIAA Paper 95-2842, July 1995.

${ }^{5}$ Tinker, M. L. "Passively Adaptive Inflatable Structure for the Shooting Star Experiment," Proceedings of the 39th Structures, Structural Dynamics, and Materials Conference, AIAA, Reston, VA, 1998, pp. 2320-2326.

${ }^{10}$ Lassiter, J., and Engberg, R., "Shooting Star Experiment Prototype Inflatable Strut/Torus Assembly Modal Survey," NASA Test Rept. SSE-DEVED97-056, NASA Marshall Space Flight Center, Huntsville, AL, June 1997

"Otto, F. (ed.), Tensile Structures, Vol. 1, MIT Press, Cambridge, MA, 1967, p. 27

${ }^{12}$ McCarty, L. H., "Inflatable Arm Segments May Lighten Shuttle's Manipulator System," Design News, Vol. 46, No. 4, 1990, pp. 150, 151.

${ }^{13}$ Leonard, R. W., Brooks, G. W., and McComb, H. G., Jr., "Structural Considerations of Inflatable Reentry Vehicles," NASA TN D-457, Sept. 1960.

${ }^{14}$ Topping, A. D., "Shear Deflections and Buckling Characteristics of Inflated Members," Joumal of Aircraft, Vol. 1, No. 5, 1964, pp. 289-293.

${ }^{15}$ Bulson, P. S., "Design Principles of Pneumatic Structures," Structural Engineering, Vol. 51, No. 6, 1973, pp. 209-215.

${ }^{16}$ Douglas, W. J., "Bending Stiffness of an Inflated Cylindrical Cantilever Beam," AlAA Journal, Vol. 7, No. 7, 1969, pp. 1248-1253.

"Steeves, E. C., "A Linear Analysis of the Deformation of Pressure Stabilized Beams," U.S. Army Natick Labs., TR USA-NLABS-TR-75-47AMEL, Natick, MA, Jan. 1975.

${ }^{18}$ Fichter, W. B., "A Theory for Inflated Thin-Wall Cylindrical Beams," NASA TN D-3466, June 1966.

${ }^{19}$ Comer, R. L., and Levy, S., "Deflections of an Inflated Circular-Cylinder Cantilever Beam," AlAA Journal, Vol. 1, No. 7, 1963, pp. 1652-1655.

${ }^{20}$ Webber, J. P. H., "Deflections of Inflated Cylindrical Cantilever Beams Subjected to Bending and Torsion," Aeronautical Journal, Vol. 86, No. 858, 1982, pp. 306-312.

${ }^{21}$ Main, J. A., Peterson, S. W., and Strauss, A. M., "Load-Deflection Behavior of Space-Based Inflatable Fabric Beams," Journal of Aerospace Engineering. Vol. 7, No. 2, 1994, pp. 225-238. 
${ }^{22}$ Hobbs, K. P., Jr., Griffith, T., Smith, S. W., and Main, J. A., "Post-Flight Testing and Analysis of Zero-G Foam Rigidized Struts," Proceedings of the 40th Structures, Structural Dynamics, and Materials Conference, AIAA, Reston, VA, 1999, pp. 2555-2561.

${ }^{23}$ Leonard, J. W., Tension Structures: Behavior and Analysis, MeGrawP.17 Hill, New York, 1988

${ }^{24}$ Main, J. A., Carlih, R. A., Garcia, E., Peterson, S. W., and Strauss, A. M., "Dynamic Analysis of Space-Based Inflated Beam Structures," Joumal of the Acoustical Society of America, Vol. 97, No. 2, 1995, pp. 10351045.

${ }^{25}$ Slade, K. N., and Tinker, M. L.. "Analytical and Experimental Investigation of the-Dynamics of-Polyimide.Inflatable.Cylinders;"-Proceedings of the 40th Structures, Structural Dynamics, and Materials Conference, AIAA, Reston, VA, 1999, pp. 2495-2506.

${ }^{26}$ Slade, K. N., "Dynamic Characterization of Thin Film Inftatable Structures," Ph.D. Dissertation, Dept. of Mechanical Engineering and Materials Science, Duke Univ., Durham, NC, 2000.

${ }^{27}$ Lassiter, J., Fischer, R., Schunk, G., Tinker, M., and Slade, K., "Multidisciplinary Testing of Thin-Film Inflatable Structures," Gossamer Spacecraft: Membrane and Inflatable Structures Technology for Space Applications, edited by C. H. M. Jenkins, Vol. 191, Progress in Astronautics and Aeronautics, AIAA, Reston, VA, 2001, pp. 333-379.

${ }^{28}$ Kilroy, K. (ed.), MSCANASTRAN Quick Reference Guide Version 70.5, MacNeal-Schwendler Corp., Los Angeles, CA, 1998.

${ }^{29}$ Blakely, K., MSCNASTRAN Basic Dynamic Analysis User's Guide, MacNeal-Schwendler Corp., Los Angeles, CA, 1993.
${ }^{30}$ Lee, S. (ed.), MSCNASTRAN Handbook for Nonlinear Analysis, MacNeal-Schwendler Corp., Los Angeles, CA, 1992.

${ }^{31}$ Lassiter, J., and Engberg, R. C., "Inflatable Strut Modal Survey Test Report," NASA Rept. SSE-DEV-ED98-070, NASA Marshal] Space Flight Center, Huntsville, AL, Aug. 1998.

${ }^{32}$ Jenkins, C. H., and Leonard, J. W., "Dynamic Wrinkling of Viscoelastic Membranes," Joumal of Applied Mechanics, Vol. 60, Sept. 1993, pp. 575582.

${ }^{33}$ Liu, X., Jenkins, C. H., and Schur, W., "Large Deflection Analysis of Pneumatic Envelopes Using a Penalty Parameter Modified Material Model," Finite Elements in Analysis and Design, Vol. 37, 2001, pp. 233-251.

${ }^{34}$ Leigh, L:-Hamidzadeh,-H.;-Tinker,-M.-L., and Slade, K. N., "Dynamic Characterization of an Inflatable Concentrator for Solar Thermal Propulsion," Proceedings of the 42nd Structures, Structural Dynamics, and Materials Conference, AIAA, Reston, VA, 2001, pp. 1678-1684.

${ }^{35}$ Smalley, K. B., Tinker, M. L., and Taylor, W. S., "Structural Modeling of a 5-m Thin Film Inflatable Antenna/Concentrator with Rigidized Support Struts," Proceedings of the 42nd Structures, Structural Dynamics, and Materials Conference, AlAA, Reston, VA, 2001, pp. 1729-1736.

${ }^{36}$ Griffith, D. T., "Experimental and Analytical Modal Analyses of an Inflated Thin Film Torus," M.S. Thesis, Dept. of Mechanical Engineering, Univ. of.Kentucky, Lexington, KY, 2000.

XXXXXX

Associate Editor 